\title{
ВАРИАЦИЯ СТОКА И ЕГО ФАКТОРОВ ${ }^{1}$
}

\author{
Н. П. Чеботарев \\ профессор, доктор технических наук \\ Воронежский государственный университет
}

Воронеж, 1949

\begin{abstract}
Аннотация: Редакция журнала «Вестник ВГУ. Серия: География. Геоэкология» публикует монографию Н. П. Чеботарева «Вариация стока и его факторов». Проблема поднятая автором в середине XX века актуальна и сегодня. Однако монография Н.П. Чеботарева стала библиографической редкостью уже сразу после выхода в свет.

Текст книги воспроизводится в авторском варианте. Для понимания важности проблемы в современных исследованиях в области гидрологии публикацию книги предваряет комментарий кандидата географических наук С.Д. Дегтярева.
\end{abstract}

Ключевые слова: речной сток, вариация стока, факторы стока.

\section{Variation of runoff and its factors}

\section{N.P. Chebotarev}

Abstract: The editorial board of the journal «Bulletin of VSU. Series: Geography. Geoecology» publishes the monograph of N.P. Chebotarev «Variation of runoff and its factors». The issue raised by the author in the middle of the 20th century is still relevant today. However, the monograph of N.P. Chebotarev became a bibliographic rarity immediately after the publication.

The text of the book is reproduced in the author's version. To understand the importance of the problem in modern research in the field of hydrology, the publication of the book is preceded by a comment by S.D. Degtyarev - candidate of geographical sciences.

Key words: river runoff, runoff variation, runoff factors.

г) Эмпирическая зависимость $C_{v x}=f(F)$

Для построения этой зависимости были вычислены коэффициенты вариации для каждого ряда речных бассейнов, находящихся в различных климатических условиях и имеющих разные площади бассейнов ЕТС. При построении зависимости $C_{v x}=F(f)$ имелось в виду получение прямой или прямых вида

$$
\lg C_{v x}=\lg B-n \lg F .
$$

Построенная, таким образом, графическая зависимость представлена на рис. 3.

По рис. 3 ясно, что между $C_{v x}$ и $F$ имеется обратная зависимость, при этом оказалось, что ко-

(С) Чеботарев Н.П., 2019

${ }^{1}$ Продолжение. Начало в журналах «Вестник ВГУ. Серия: География. Геоэкология» №3/2018 г., №4/2018 г. и № $1 / 2019$ г.

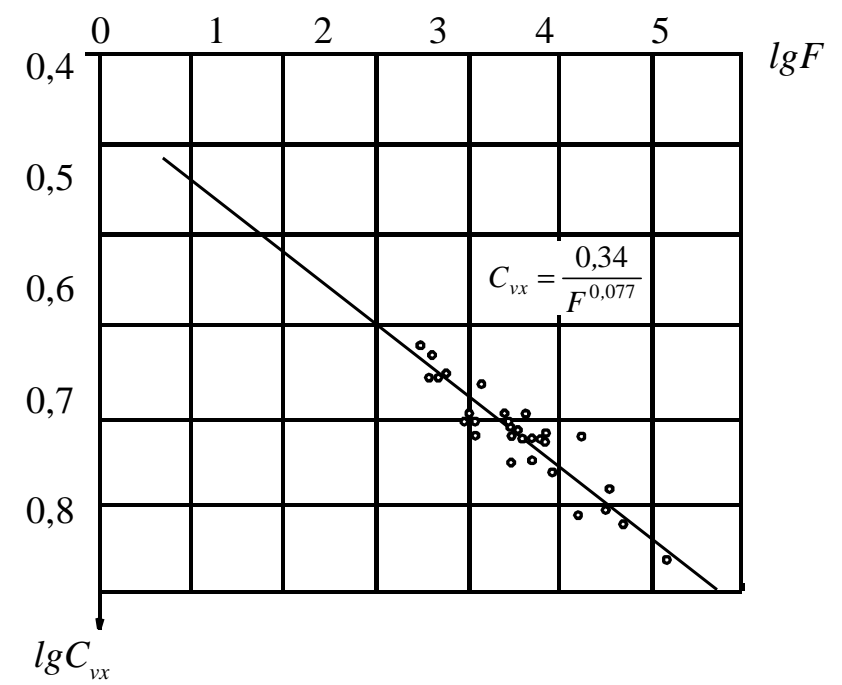

Puc. 3. График связи $\lg C_{v x}=f(\lg F)$ 
эффициент корреляции между этими величинами $r=-0,848 \pm 0,048$, а уравнение регрессии имеет вид:

$$
\lg C_{v x}=-0,077 \lg F-0,468 .
$$

Средняя величина ошибки связи $\Delta \lg C_{v x}=+0,03$. Потенцируя уравнение (40), найдем, что

$$
C_{v x}=\frac{0,34}{F^{0,077}} .
$$

Полученная формула (41) может служить для практического применения, когда необходимо установить $C_{v x}$. Формула (41) дает среднее отклонение $=4,48 \%$, при наибольшем отклонении (один случай $=12,7 \%$ ).

Обращаем внимание на показатель степени при $F$ формуле (41) - он близок к показателю при $F$, существующих формул для $C_{v y}$, а именно, по I формуле Крицкого и Менкеля =0,083. По II формуле Кри цкого и Менкеля $n=0,060$, по 2-й формуле Антон ов а $n=0,076$.

Относительно малое расхождение в приведенных значениях показателей степени $\mathrm{n}$ с показателем, полученным мной, указывает на один и тот же источник причин закономерности связей как между $C_{v y}$ и $F$, так и между $C_{v x}$ и $F$.

Остановим теперь свое внимание на параметpe $B=0,34$ формулы (41). Какова физическая сущность этого параметра? Подставляя в уравнение (36) $F=I$, устанавливаем, что $B=C_{v x}$, т.е. параметр $B$ - это есть коэффициент вариации при $F=I$. C другой стороны выше было установлено, что по своему физическому смыслу значение параметра В соответствует значению $C_{v x(i)}$, т.е. что параметр В представляет собой среднее значение коэффициента вариации осадков, найденного для площади бассейна, равной одному квадратному километpy. Значения параметров $B=C_{v x(i)}$ колеблются в пределах от 0,31 до 0,38. Это колебание вызывается неоднородностью метеорологических условий отдельных бассейнов рек, вызывающих асинхронность в колебаниях годовых высот осадков. При этом, чем площадь больше, тем больше должна быть эта асинхронность и тем меньше значение $C_{v x}$ по отношению к $C_{v x(i)}$. Поэтому, сравнивая отношение $\frac{C_{v x(i)}}{C_{v x}}$ с площадями бассейнов, мы нашли, что такое отношение возрастает с увеличением площади бассейна. Отношение $\frac{C_{v x(i)}}{C_{v x}}$ возрастает от 1,88 при $F=3738$ до 2,96 при $F=1.354 .270$.
Отношение $\frac{C_{v x(i)}}{C_{v x}}$ показывает, во сколько раз происходит понижение значения $C_{v x(i)}$ в силу неоднородности и неравномерности в метеорологических условиях бассейна. При $F=I$ это отношение равно единице, т.е. при этой площади бассейна не происходит понижение $C_{v x(i)}$. Очевидно в последнем случае метеорологические условия являются однородными или близкими к этому.

Теперь остается выяснить еще один вопрос. Можно ли параметр $B=C_{v x(i)}$ получить на основе непосредственных наблюдений? На этот вопрос можно было бы дать, казалось, вполне определенный и положительный ответ, а именно: каждая метеорологическая станция, а точнее каждый дождемер может дать значение годовых высот осадков с помощью которых можно вычислить $C_{v x(i)}$. Приведем для иллюстрации вычисленные значения $C_{v x(i)}$ для отдельных метеорологических станций, найденных за продолжительный период времени. Среднее значение $C_{v x(i)}$ для 51 пункта составляет $=0,200$. Для местности от $40^{\circ}$ до $50^{\circ}$ широты среднее значение $C_{v x(i)}=0,224$; для $50^{\circ}-60^{\circ}$ широты $C_{v x(i)}=0,175$ и для $60^{\circ}-65^{\circ}$ широты $C_{v x(i)}=0,188$. Из этого можно заключить, что в широтном отношении $C_{v x(i)}$ изменяется сравнительно мало.

Высокие значения встречаются как на юге, так и на севере, хотя с более высоким значением $C_{v x(i)}$ встречается на юге чаще. Максимальное значение для широты до $50^{\circ} C_{v x(i)}=0,306$ (Таганрог), а для широты от $60^{\circ}$ до $65^{\circ} C_{v x(i)}=0,257$ (Н. Ладога). Минимальное значение $C_{v x(i)}$ до $50^{\circ}$ широты встречается $=0,154$ (Тбилиси), а для широты от $60^{\circ}$ до $65^{\circ}$ $C_{v x(i)}=0,063$ (Кемь).

Для субтропических и тропических областей значения $C_{v x(i)}$ будут выше, так как изменчивость осадков в этих областях выше, чем в более высоких широтах. По этому вопросу проф. В. Кеппен «Основы климатологии» пишет: «Это, очевидно, зависит от того, что в тропиках, даже в расположенных близко местах, на одной и той же высоте над морем, наряду с большим однообразием температурных условий создаются гораздо более разнообразные условия для выпадения осадков, чем в более высоких широтах. Если это обстоятельство является следствием постоянства направления ветpa a тропиках, благодаря чему каждый горный хребет имеет свою надветренную сторону, то случайные отклонения дождливых и сухих периодов, со своей стороны, происходят вследствие случайных перемещений системы ветров и связанных с последней «дождливых и сухих областей». 


\begin{tabular}{|l|l|c|c|c|c|}
\hline $\begin{array}{c}\text { №№ } \\
\text { п/п }\end{array}$ & Название пункта & $C_{v x}$ & $\begin{array}{c}\text { Площадь } \\
f \text { км }^{2}\end{array}$ & $\begin{array}{c}\text { Расстоян. } \\
1 \text { км }\end{array}$ & $\begin{array}{c}\text { Радиус } \\
R \text { км }\end{array}$ \\
\hline 1 & Таганрог & 0,306 & 3,89 & 1,97 & 1,11 \\
2 & Астрахань & 0,289 & 8,13 & 2,85 & 1,61 \\
3 & Ялта & 0,286 & 9,55 & 3,09 & 1,74 \\
4 & Ставрополь & 0,224 & 214 & 14,6 & 8,25 \\
5 & Харьков & 0,216 & 355 & 18,8 & 10,6 \\
6 & Среднее (по 51 пун.) & 0,200 & 1000 & 31,6 & 17,8 \\
7 & Ряжск & 0,187 & 2344 & 48,4 & 27,3 \\
8 & Москва & 0,152 & 34670 & 186 & 105 \\
9 & Ленинград & 0,147 & 53700 & 231 & 130 \\
10 & Кола & 0,139 & 107200 & 327 & 185 \\
\hline
\end{tabular}

Сравнивая полученные значения для $C_{v x(i)}$, непосредственно вычисленные для отдельных станций, с данными, полученными по формуле (41), мы видим, что последние имеют значения значительно выше. Это сравнение показывает, что вычисленные $C_{v x(i)}$ по данным станциям являются преуменьшенными и, следовательно, на вопрос, поставленный выше, нужно ответить отрицательно. Но чем же объяснить такое сильное расхождение в значениях $C_{v x(i)}$, полученных различными путями? Такое расхождение, надо думать, получается от того, что каждая станция, или точнее каждый дождемер, по своим данным, характеризует режим выпадающих осадков не в одной только той точке, в которой он стоит, а некоторой площади, расположенной вокруг него, т.е. он дает некоторые серединные данные, характерные для этой площади. Величина последней в среднем при $C_{v x(i)}=0,2$ (по фор. 41) может достигать 1000 км² $^{2}$.

д) О возможных предельных расстояниях между дождемерами

Из предыдущих рассуждений следует, что чем меньше площадь бассейна, тем больше средний коэффициент корреляции $r_{x}$. Этим положением мы установили, что $r_{x}$ характеризует влияние размера площади бассейна на $C_{v x}$ и что по той же причине $C_{v x}$ находится в обратной зависимости от площади бассейна. Результаты исследований этих зависимостей позволили затронуть весьма актуальный вопрос - о предельных расстояниях между дождемерами.

Предположим, имеется некоторая площадь $F$ с однородной поверхностью в отношении физико-географических условий (растительность, рельеф, и т.д.). На каком расстоянии друг от друга следует устанавливать дождемеры, чтобы вся пло- щадь могла быть, с достаточной полнотой и точностью для практики, охарактеризована в отношении режима осадков? Для разрешения поставленного вопроса необходимо найти тот размер площади, который бы вполне мог быть охарактеризован наблюдениями одной станции. Назовем эту площадь «площадью сферы влияния» дождемера и обозначим ее через $f$. Очевидно, что если будем знать $f$, то будем знать и расстояния между дождемерами $I$ и радиус площади сферы влияния.

Рассматривая формулу

$$
C_{v x}=\frac{C_{v x(i)}}{F^{n}},
$$

мы видим, что $C_{v x(i)}$ - максимальное значение $C_{v x}$ при $F=I$. Для одиночных дождемеров $C_{v x}$ колеблется от 0,06 до 0,306 и в среднем составляет $=0,200$.

При среднем значении $C_{v x}=0,200$ площадь сферы влияния $f=1000 \mathrm{kм}^{2}$. Из (41) мы видим, что $f$ зависит от коэффициента вариации осадков, наблюдаемых отдельными дождемерами.

Приведем данные, для: $f$, и расстояния $I$, а также для радиуса круга равновеликого - площади $f$ 9 пунктов ЕТС, лежащих в различных широтных условиях (табл. 3).

Из данных, приведенных в табл. 3, следует, что расстояния между дождемерами могут колебаться от 2 до 327 км и в среднем для 51 пункта $I_{c p}=32$ км.

Идя теми же путями, можно придти к предельным расстояниям и по отношению к другим метеорологическим элементам.

\section{3. ЗАВИСИМОСТЬ КОЭФФИЦИЕНТА ВАРИАЦИИ ГОДОВЫХ ВЫСОТ ОСАДКОВ ОТ НОРМЫ РАСХОДА АТМОСФЕРЫ}

Представим себе данную площадь бассейна, разделенную на отдельные части. Пусть для каж- 
дой части известны площадь ее $F_{i}$ и высота осадков $x_{i}$, тогда средними высотами осадков для всего бассейна

$$
\overline{x_{0}}=\frac{F_{1} \overline{x_{1}}+F_{2} \overline{x_{2}}+\ldots+F_{n} \overline{x_{n}}}{F_{0}} .
$$

Обозначив произведение $F_{i} x_{i}$ через $N_{i}$, равенство (42) можно переписать так:

$$
\overline{N_{0}}=\overline{N_{1}}+\overline{N_{2}}+\ldots+\overline{N_{n}} .
$$

Ограничим наше исследование двумя частями площади, тогда

$$
\overline{N_{0}}=\overline{N_{1}}+\overline{N_{2}} \text {. }
$$

Пользуясь методами математической статистики, можно получить равенство (44), выраженное через стандарты, а именно:

$$
\begin{gathered}
\sigma_{0}{ }^{2}=\sigma_{1}{ }^{2}+\sigma_{2}{ }^{2}+2 \sigma_{1} \sigma_{2} r_{1 / 2}, \\
\sigma_{1}{ }^{2}=\sigma_{0}{ }^{2}+\sigma_{2}{ }^{2}-2 \sigma_{0} \sigma_{2} r_{1 / 2} .
\end{gathered}
$$

Так как

$$
\sigma_{1}=\overline{N_{1}} C_{v 1}, \sigma_{0}=\overline{N_{0}} C_{v 0} \text { и } \sigma_{2}=\bar{N} C_{v 2},
$$
TO

$$
{\overline{N_{1}}}^{2} C_{v 1}{ }^{2}={\overline{N_{0}}}^{2} C_{v 0}{ }^{2}+{\overline{N_{2}}}^{2} C_{v 2}{ }^{2}-2 \overline{N_{0}} C_{v 0} \overline{N_{2}} C_{v 2} r_{0 / 2} .
$$

Принимая во внимание, что $r_{0 / 2} \frac{C_{v 2}}{C_{v 0}} \cong 1$ (это хорошо доказывается на отдельных примерах, например, бассейн р. Свири и др.), получим:

$$
{\overline{N_{1}}}^{2} C_{v 1}{ }^{2}-{\overline{N_{2}}}^{2} C_{v 2}{ }^{2}={\overline{N_{0}}}^{2} C_{v 0}{ }^{2}-2 \overline{N_{0}} \overline{N_{2}} C_{v 0}{ }^{2} \text {, }
$$

откуда

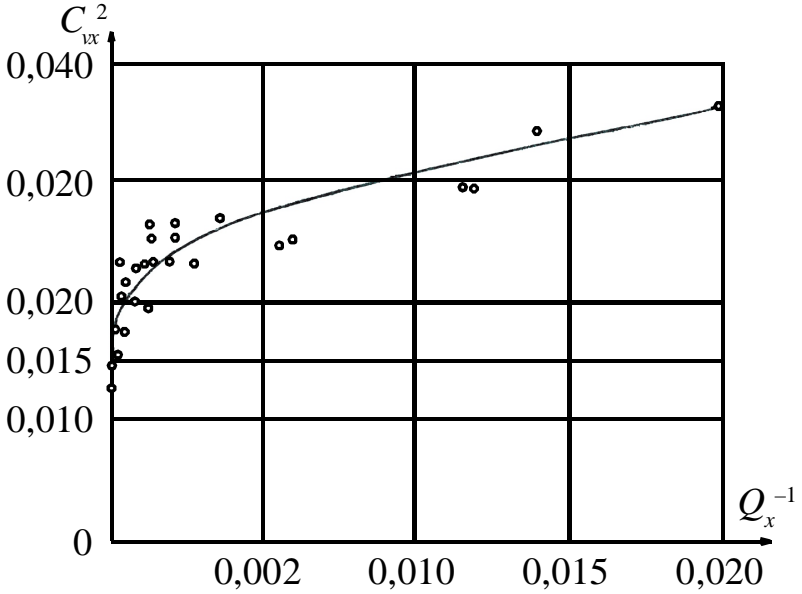

Puc. 4. График зависимости $C_{v x}{ }^{2}=f\left(Q_{x}^{-1}\right)$
Применяя прием Ефимовича (21) к случаю преобразования подобного равенства, предложенный им в отношении годового стока, можно доказать, что

$$
C_{v x}=\sqrt{B_{1}(x F)^{-1}+D},
$$

или

$$
C_{v x}=\sqrt{B_{2} Q_{x}^{-1}+D},
$$

где $Q_{x}=\frac{\overline{x_{0}} F}{31536}-$ расход атмосферы,

$\overline{x_{0}}$ - норма осадков в мм,

$F$ - площадь бассейна в км ${ }^{2}$.

Зависимость между $C_{v x}{ }^{2}$ и $Q_{x}^{-1}$ представлена на основе наблюденных данных в графическом виде (рис. 4).

Из рисунка ясно, что эту зависимость можно представить в виде параболической кривой, которая аналитически выражается так:

$$
C_{v x}{ }^{2}=\mathrm{A}\left[Q_{x}^{-1}\right]^{\mathrm{n}},
$$

или, извлекая квадратный корень из обеих частей равенства, получим:

$$
C_{v x}=\sqrt{A}\left[Q_{x}^{-1}\right]^{n / 2}=\frac{A_{1}}{Q_{x}^{n_{1}}},
$$

где

$$
\sqrt{A}=A_{1} \text { и } \frac{n}{2}=n_{1} .
$$

Параметры $A_{1}$ и $n_{1}$ были найдены с помощью графически построенной функции $2 \lg C_{v x}=f\left(\lg Q_{x}^{-1}\right)$ (рис. 5).

После чего уравнение (53) с найденными числовыми выражениями параметров будет окончательно иметь следующий вид:

$$
C_{v x}=\frac{0,25}{Q_{x}^{0,083}} .
$$

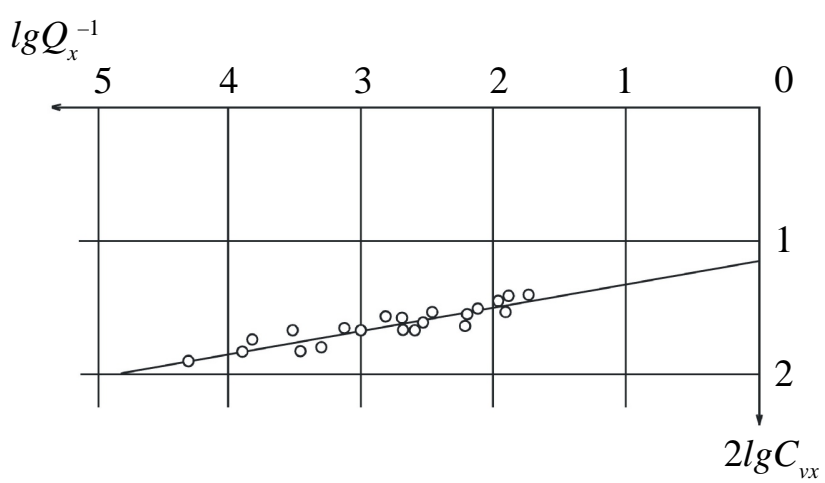

Puc. 5. График связи $2 \lg C_{v x}=f\left(\lg Q_{x}^{-1}\right)$ 
Произведенная проверка для тех же 32 речных бассейнов дала среднее отклонение $= \pm 4,6 \%$, при максимальном одном значении $14 \%$. Коэффициент корреляции между $2 \lg C_{v}$ и $\lg Q_{x}^{-1}$ составляет $=0,83$.

В формуле (53) $Q_{x}$ можно заменить его значением $=\frac{\overline{x_{0}} F}{31536}$ или вообще оно может быть принято равным $\xi \overline{x_{0}} F$, где $\xi-$ переводный коэффициент, тогда

$$
C_{v x}=\frac{A_{1}}{\left(\xi \overline{x_{0}} F\right)^{n_{1}}} .
$$

Разделив числитель и знаменатель правой части на $\left(\xi \overline{x_{0}}\right)^{n_{1}}$ получим:

$$
C_{v x}=\frac{A_{1} /\left(\xi \overline{x_{0}}\right)^{n_{1}}}{F^{n_{1}}}=\frac{C_{v x(i)}}{F^{n_{1}}},
$$

где

$$
C_{v x(i)}=\frac{A_{1}}{\left(\xi \overline{x_{0}}\right)^{n_{1}}} .
$$

Из (56) следует, что одной из причин колебания $C_{v x(i)}$ являются колебания значений $\overline{x_{0}}$, с которыми $C_{v x(i)}$ находится в обратной зависимости.

Физический смысл параметра $A_{1}$ вытекает из последнего выражения для $C_{v x(i)}$, когда $\left(\xi \overline{x_{0}}\right)=1$ или когда $Q_{x}=1$ в формуле (54). 